\title{
Characterization and Analyses of hydrophobic clusters, acetylation and myristoylation sites in plant glutathione peroxidase sequences
}

\author{
Sayak Ganguli ${ }^{1, *}$, Anisha Polley $^{2}$, Abhijit Datta $^{3}$ \\ ${ }^{1}$ DBT Centre for Bioinformatics, Presidency University, Kolkata, India \\ ${ }^{2}$ Department of Biological Sciences, Presidency University, Kolkata, India \\ ${ }^{3}$ Department of Botany, Jhargram Raj College, Jhargram, West Bengal, India \\ *E-mail address: sayakbif@yahoo.com
}

\begin{abstract}
Incomplete reduction of oxygen molecules is the primary source for the formation of reactive oxygen species (ROS) during cytosolic metabolism or mitochondrial respiration. These phenomenons may be as a result of biotic or abiotic stress. Exposure to exogenous stimuli such as radiation might be an alternative pathway of ROS production. Thus plants require counter defense strategies to combat the increase of this toxic molecular build up in its cell cytoplasm. As a result they have devised an army of free radical scavenging enzymes which enable them to dissipate the oxidative stress imposed by the accumulation of these toxic moieties. Glutathione Peroxidase forms an important part of this arms race along with several catalases and organelle specific enzymes such as superoxide dismutase. Plant glutathione peroxidases(GPXs) have been studied exclusively for their evolutionary lineages since they represent a hybrid class of molecules in context of the presence and absence of selenocysteine at their catalytic centres, the former situation predominant in non vascular plant groups while the later a predominant feature of vascular plants. This analysis focuses on three important aspects of protein structure analyses - hydrophobic cluster analyses for identification of homologues, and acetylation and myristoylation sites which provide us with information regarding the post translational modifications of a particular protein group. Specific patterns of clusters along with acetylation and myristoylation site frequencies were obtained which indicate that GPXs of non vascular plant members possess less chances of getting myristoylated while acetylation was predominant in most land plant lineages but absent in aquatic members.
\end{abstract}

Keywords: Plant glutathione peroxidase; post transcriptional modification; protein structure prediction; myristoylation

\section{INTRODUCTION}

Reactive oxygen species (ROS) such as superoxide, $\mathrm{H}_{2} \mathrm{O}_{2}$ and lipid hydroperoxides are toxic cellular metabolites which are rapidly detoxified by various enzymatic and nonenzymatic scavengers. At the same time, they serve as signaling molecules regulating important biological processes in both animal and plant cells (Halliwell 2006). In plants, they have been shown to control biotic and abiotic stress responses, programmed cell death, hormone signaling, cellular differentiation, growth and development (Potikha et al. 1999, 
Mittler et al. 2004, Gechev et al. 2006). Thus, the ROS-scavenging systems need to maintain a redox homeostasis, above which the different signals can be detected. In many cases, thiolcontaining proteins serve as transducers, which are either activated or inactivated in response to ROS (Foyer and Noctor 2005). A major candidate as a ROS-level regulator is the phospholipid hydroperoxide glutathione peroxidase (PHGPx) which serves as a detoxifying and signaling enzyme.

Glutathione peroxidases are an important group of plant enzymes having a very large substrate spectrum. They almost occur ubiquitously in plant cells - in the cytosol, mitochondria, chloroplast as well as in the endoplasmic reticulum. The main reaction that they catalyze is the reduction of hydrogen peroxide and some lipid hydroperoxides where glutathione acts as the reductor. Since the report of the first glutathione peroxidase isolated from Nicotiana species by Criqui et.al. (1992) there has been a significant number of sequences that have made their way in public repositories such as Genbank and Uniprot. Gaber et.al. (2012) has cloned eight glutathione peroxidase genes in Arabidopsis. Holland et.al. (1993), Jung et.al. (2002) and Margis et.al. (2008) have successfully classified the existing sequences into five major groups: Clades I and II: Chloroplastic and cytosolic isoforms respectively; Clades III and IV: cytosolic and secreted proteins; Clade V: cytosolic protein with $\mathrm{N}$ terminal transit peptides.

Recently we have demonstrated through the machine learning approach of Kohonen Map that plant glutathione peroxidases clearly can be clustered into four major groups based on their sequence composition and structural organization (Ganguli and Datta 2014). Despite several successful sequence submissions, very few crystallographic structures of the enzyme is available in public repositories. At the time of manuscript preparation there were only three hits for plant glutathione peroxidases in PDB; two belonging to poplar and the other to Arabidopsis thaliana. (Fig. 1).

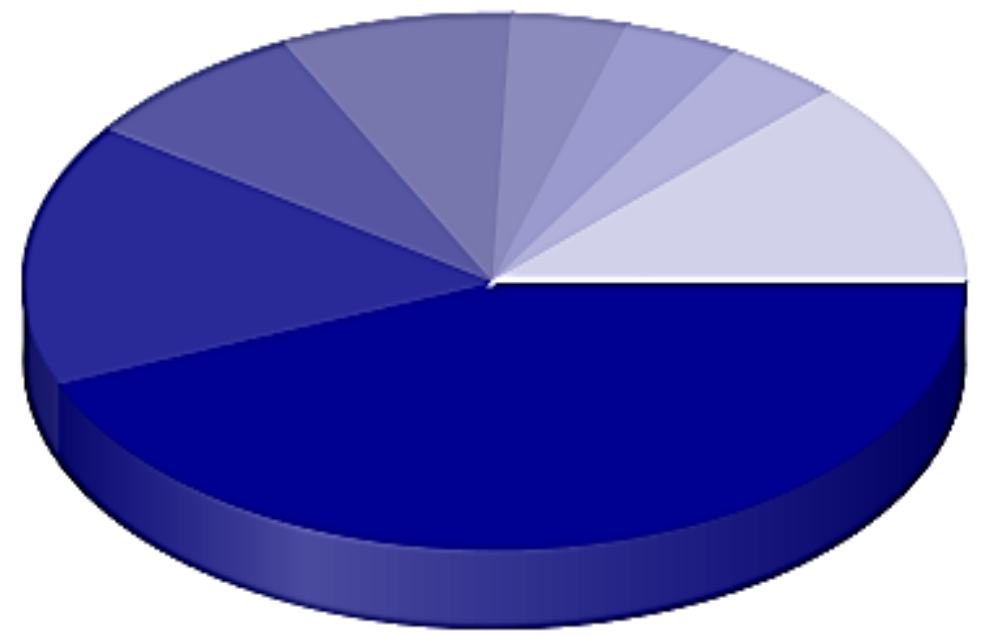

$44 \%$ Homo sapiens (11 hits)

$16 \%$ Trypanosoma brucei (4 hits)

$8 \%$ Schistosoma mansoni (2 hits)

$8 \%$ Populus trichocarpa $\times$... (2 hits)

$4 \%$ Mus musculus ( 1 hits)

4\% Trypanosoma cruzi (1 hits)

$4 \%$ Saccharomyces cerevisiae (1 hits)

$12.5 \%$ Other (3 hits)

Fig. 1. Distribution of glutathione peroxidase structures in PDB.

Thus a concerted effort is required for successful evaluation and understanding of the actual reaction mechanisms of interactions of this group of enzymes for future metabolic engineering and designing of advanced proteins. 
Hydrophobic cluster analyses was initially designed to detect remote homology. However, this property can be utilized to properly identify and classify a homologous set of proteins as well. Acetylation is the most common type of covalent modification found in plants. There is still no universal consensus motif for $\mathrm{N}$-terminal acetylations. The presence of numerous $\mathrm{N}$ acetyl transferases is evidence in itself that the acetylated termini are diverse and complex in their sequence composition. This process can modify protein function, proteinprotein interaction, and/or thermal stability (Pesaresi et.al. 2003). Nearly $2 \%$ of all plant proteins are $\mathrm{N}$ - Myristoylated and this has led the irreversible lipid modification to be established as one of the key plant post translational modification in plants. The Nmyristoylome of Arabidopsis indicates that a large number of myristoylated proteins are involved in control and repair of environmental damage. The reaction is catalyzed by the Nmyristoyltransferase (NMT). Several reports also suggest that membrane proteins are frequently myristoylated in an effort to control plant immune pathways. Traverso et.al. (2008) indicate that myristoylated proteins mostly are involved in plant stress signaling pathways.

In an effort to understand and elucidate the structural complexities of plant glutathione peroxidases, an extensive exploration of its sequence based functional properties namely hydrophobic clusters, $\mathrm{N}$ terminal acetylation and $\mathrm{N}$ - myristoylation were explored in this work.

\section{MATERIALS AND METHOD}

Hydrophobic cluster prediction was performed using the method described by Gaboriaud et al where the sequence duplication allows the reader to immediately identify all six residues surrounding any given sequence position.

A neural network based approach was devised for the prediction of the sites for acetylation and myristoylation; where the predictor score $S$ is based on a result of trained artificial neural networks. The range of negative to positive hits fall within: 0.011067 to 0.988849 (average responses are between 0 and 1). The score $S$ is defined as: $S=$ Positive Negative. A score close to one involves a high confidence for acetylation or myristoylation, whereas a score close to -1 is a strong decision in favour of the absence of acetylation or myristoylation.

The Confidence intervals used for the study were as follows:

- $<S<0.4--->$ Low Confidence

- $0.4<S<0.85-->$ Medium Confidence

- $0.85<S<1$--- $>$ High Confidence

\section{RESULTS AND DISCUSSION}

Hydrophobic clusters have been established as markers of regular secondary structures. Thus they can be used for the identification of homologous folds. Two basic types of clusters were identified - simple clusters with prominent secondary structure propensity and simple clusters devoid of any prominent secondary structure propensity. 


\section{TYPES OF IDENTIFIED HYDROPHOBIC CLUSTERS IN PLANT GLUTATHIONE PEROXIDASE SEQUENCES}

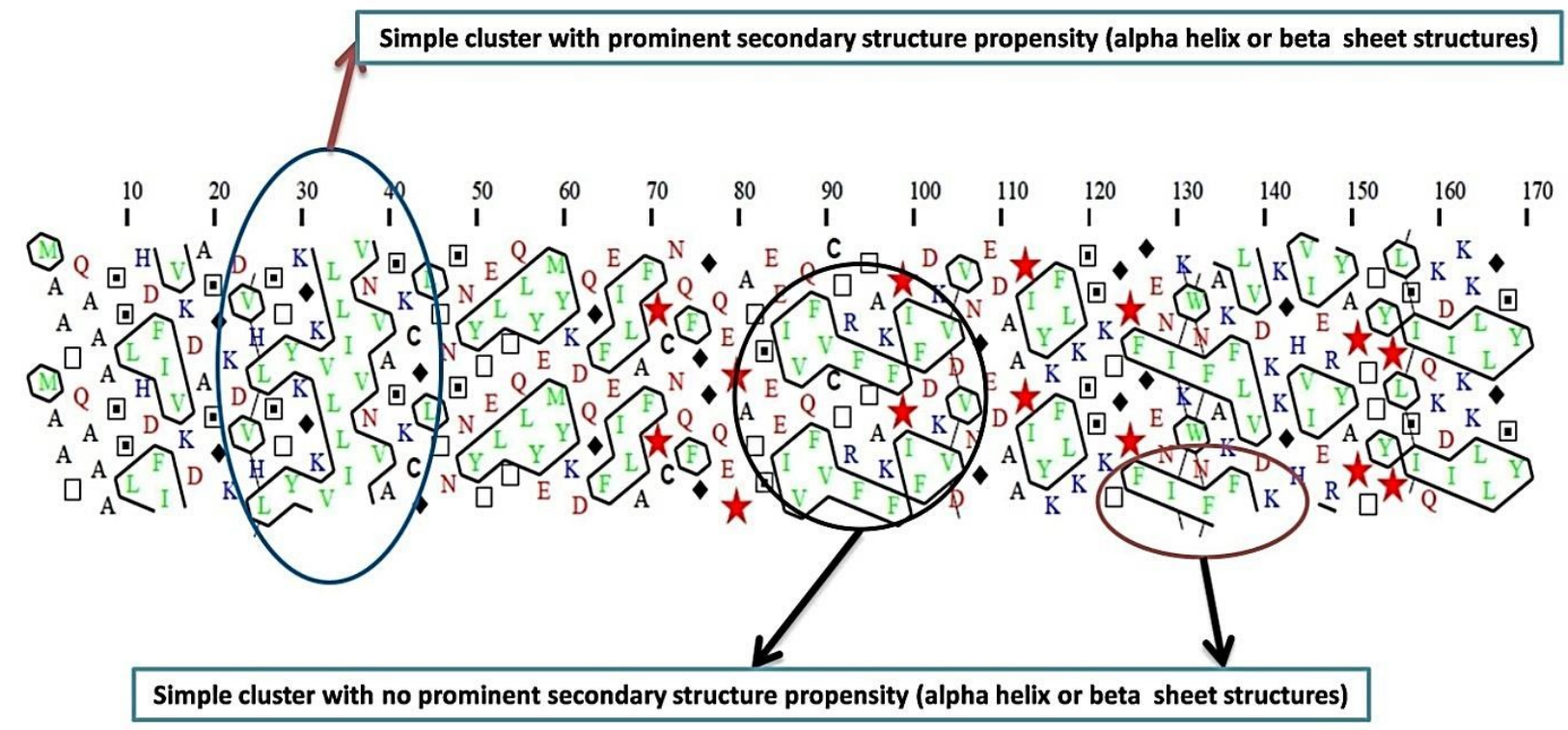

\section{DIFFERENT SYMBOLS USED:}

Prolines $\downarrow$ and Glycines which are often present in loops

Cysteines $\square$ which may be involved in disulfide bonds.

Fig. 1. Results of hydrophobic cluster analyses (Full results can be obtained from the corresponding author as a zipped archive).

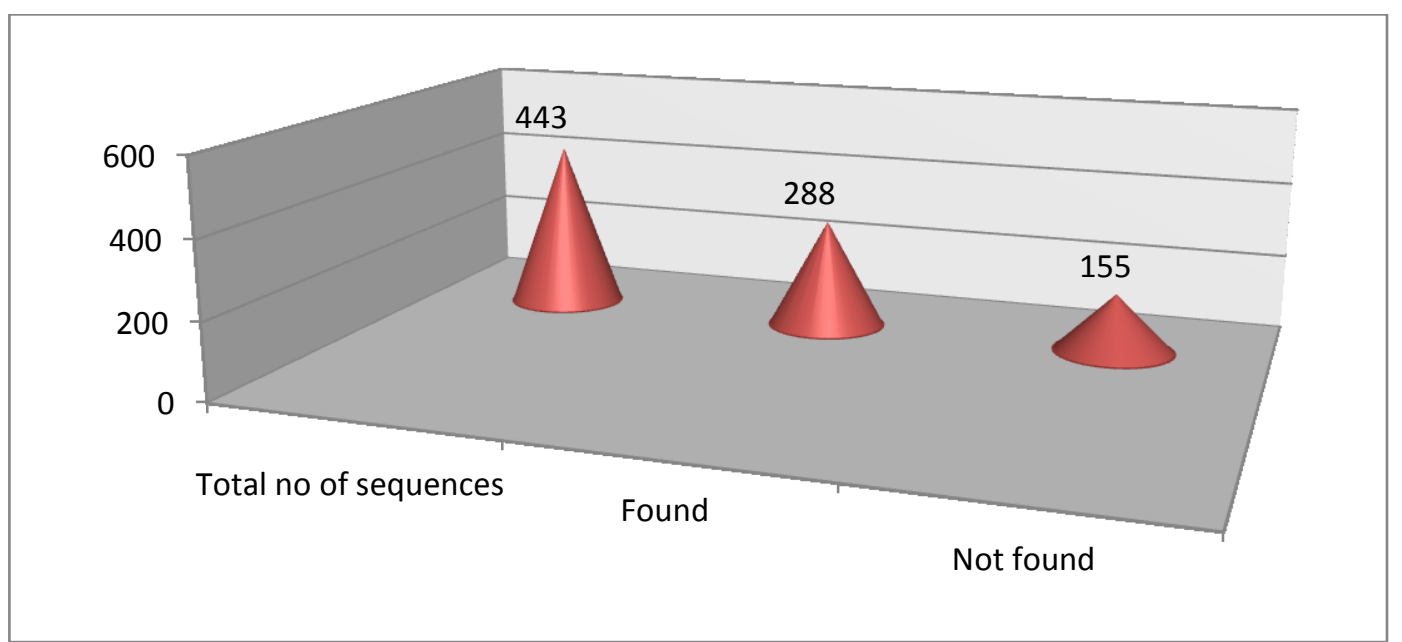

Fig. 2. The frequency of acetylation in the sequences under study. 


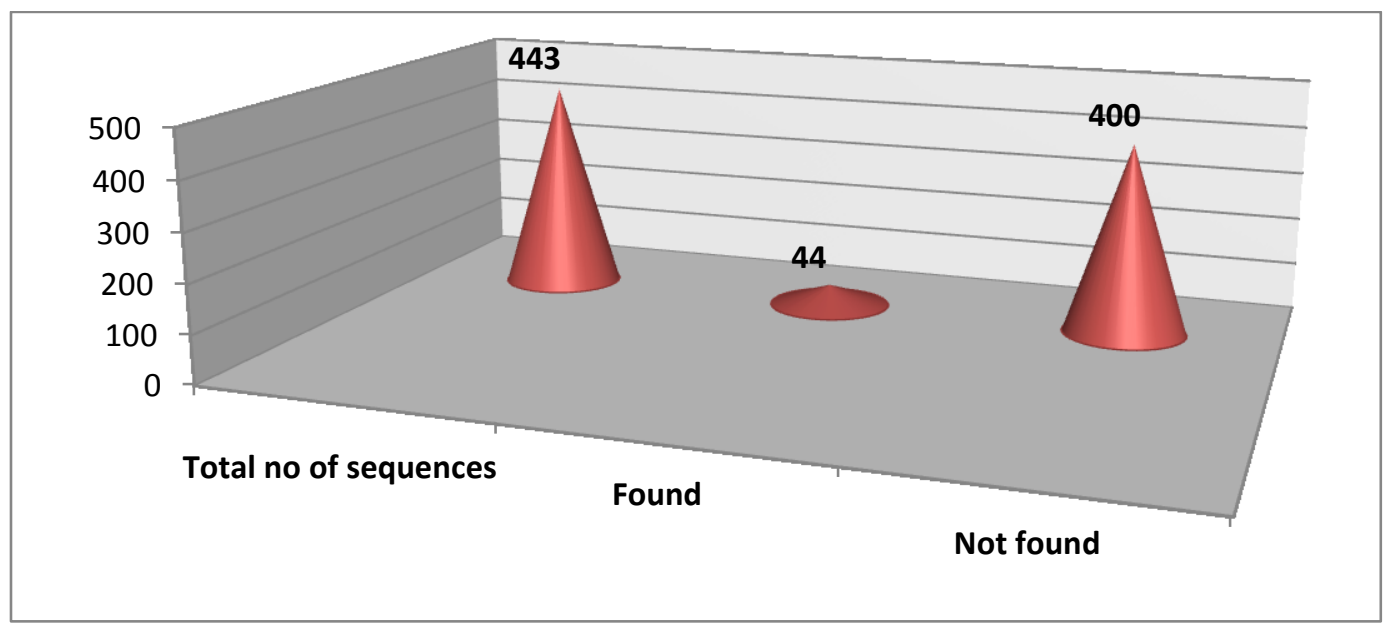

Fig. 3. The frequency of myristoylation in the sequences under study.
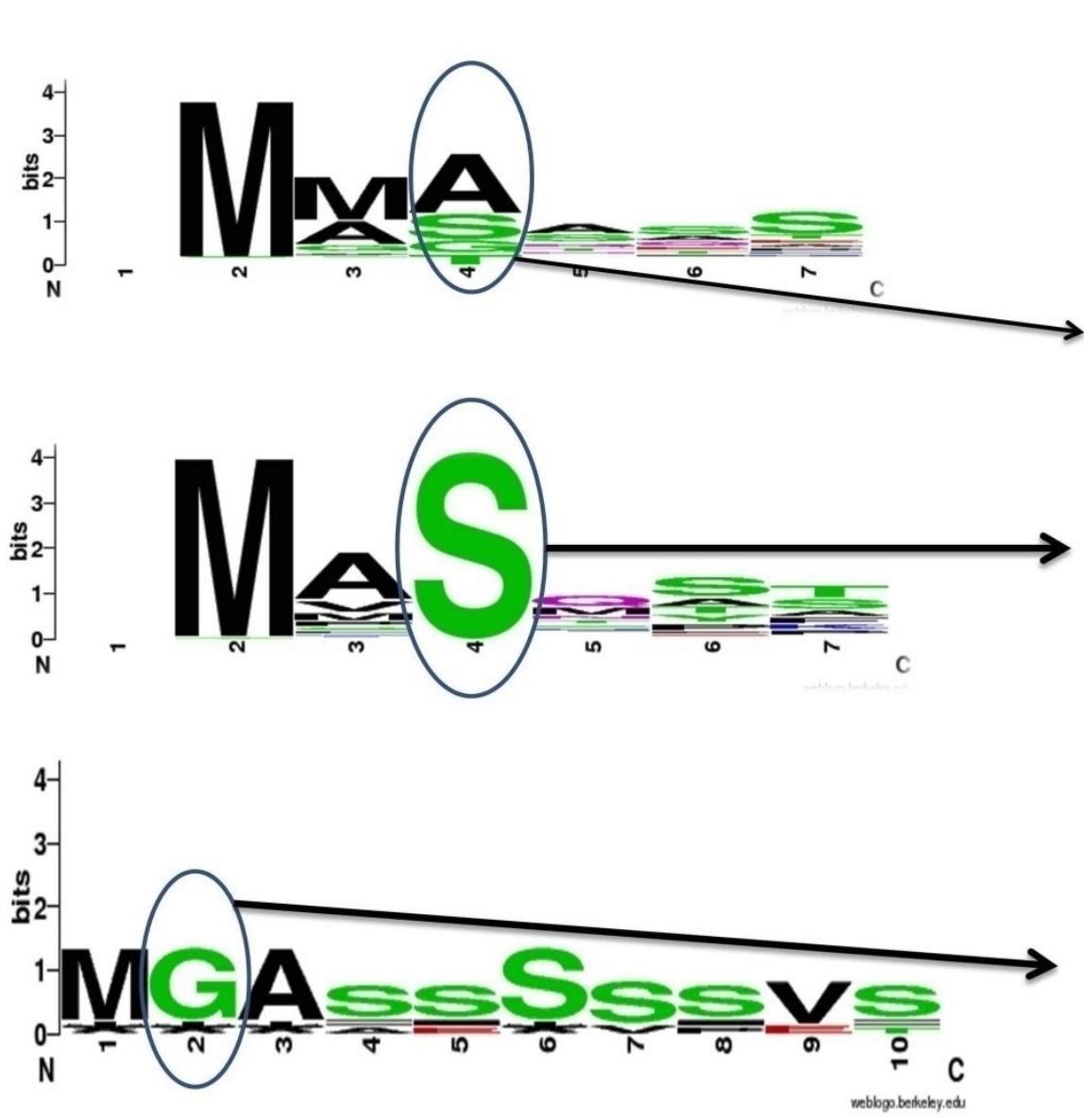

Sequence Logo to detect residue propensity at the $\mathrm{N}$ Terminal region of plant GpXs.

Upper Panel: MSA generated logo for all sequences used for the study

Middle Panel: MSA generated logo for Sequences which have positive $\mathbf{N}$ terminal ACETYLATION residues dominated by serine residue at fourth position

Lower Panel: MSA generated logo for Sequences which have positive $\mathbf{N}$ terminal MYRISTOYLATION residue $\left(2^{\text {nd }}\right.$ position glycine)

Fig. 4. Residue propensities for $\mathrm{N}$ terminal acetylation and myristoylation in plant glutathione peroxidase sequences. 
Protein acetylation is influenced by the cellular level of both acetyl-CoA and NAD ${ }^{+}$, and regulates the activity of several enzymes. Acetyl-CoA is thus ideally placed to act as a key molecule linking the energy balance of the cell to the regulation of gene expression and metabolic pathways via the control of protein acetylation. Here $53 \%$ of the total 443 sequences of GPx are acetylated with their desired sites. Despite reports that plant stress proteins have a higher probability of myristoylation, the probability of sequences getting myristoylated were low, with only $10.75 \%$ of all sequences exhibiting propensity of getting myristoylated.

The results obtained in the study indicate towards proper functional conservedness in glutathione peroxidase sequences with specific patterns of hydrophobic clusters present in all the sequences under study. Probability of $\mathrm{N}$ terminal acetylation was found to be concentrated at a serine residue occurring in the fourth position of the amino acid sequences of the proteins under study while all the proteins showing propensity for $\mathrm{N}$ - myristoylation possessed a glycine residue in the second position. These sites should be investigated further for their possible roles in the interactions with key substrates and in silico mutagenesis studies can be performed for evaluating their necessity in protein structure maintenance and function.

\section{CONCLUSION}

Several hydrophobic clusters, myristoylation sites and acetylation sites were identified and analyzed. Specific residue propensities were identified for both the post translational modification events. These sites can be further used for experimental protocols concerning protein function and design of metabolically active peptides.

\section{Acknowledgements}

The authors acknowledge the support of the BTBI - scheme of Department of Biotechnology (DBT), Government of India, for maintenance of the facility.

\section{References}

[1] Beeor-Tzahar T., Ben-Hayyim G., Holland D., Faltin Z., Eshdat Y., FEBS Lett. 366 (1995) 151-155.

[2] Callebaut I., Labesse G., Durand P., Poupon A., Canard L., Chomilier J., Henrissat B, Mornon JP., Cell Mol Life Sci 53 (1997) 621-645.

[3] Chothia C., Lesk AM., EMBO J 5 (1986) 823-826.

[4] Edwards R., Physiol Plant 98 (1996) 594-604.

[5] Eshdat Y., Holland D., Faltin Z., Ben-Hayyim G., Physiol. Plant. 100 (1997) 234-240.

[6] Foyer C.H., Noctor G., Plant Cell 17 (2005) 1866-1875.

[7] Gaboriaud C, Bissery V, Benchetrit T, Mornon JP. (1987). FEBS Lett ;224:149-155

[8] Ganguli S., Datta A., Annual Research \& Review in Biology 24(4) (2014) 3810-3815.

[9] Gechev T.S., Van Breusegem F., Stone J.M., Denev I., Laloi C., Bioessays 28 (2006) 1091-1101. 
[10] Gueta-Dahan Y., Yaniv Z., Zilinskas BA., Ben-Hayyim G., Planta 203 (1997) 460-469.

[11] Halliwell B., Plant Physiol. 141 (2006) 312-322.

[12] Herbette S., Roeckel-Drevet P., Drevet J.R., FEBS J. 274 (2007) 2163-2180.

[13] Jung B.G., Lee K.O., Lee S.S., Chi Y.H., Jang H.H., Kang S.S., J. Biol. Chem. 277 (2002) 12572-12578.

[14] Navrot N., Collin V., Gualberto J., Gelhaye E., Hirasawa M., Rey P., et al., Plant Physiol. 142 (2006) 1364-1379.

[15] Pesaresi P, Gardner NA, Masiero S, Dietzmann A, Eichacker L, Wickner R, Salamini F, Leister D., Plant Cell 15 (2003) 1817-1832.

[16] Pierre M, Traverso JA, Boisson B, Domenichini S, Bouchez D, Giglione C, Meinnel T., The Plant Cell 19 (2007) 2804-2821.

[17] Potikha T.S., Collins C.C., Johnson D.I., Delmer D.P., Levine A., Plant Physiol. 119 (1999) 849-858.

[18] Sabeh F., Wright T., Norton S.J., Enzyme Protein 47 (1993) 92-98.

[19] Ursini F., Maiorino M., Roveri A., Biomed. Environ. Sci. 10 (1997) 327-332.

[20] Willekens H, Chamnongpol S, Davey M, Schraudner M, Langebartels C, Van Montagu M, Inz e D, Van Camp W., EMBOJ 16 (1997) 4806-4816. 